\title{
Nodal Recurrence as Primary Driver of Early Relapse in Patients with SLN-Positive Melanoma: What Does It Mean for Providers and Patients?
}

\author{
Nellie E. Farrow, MD, Kristen E. Rhodin, MD, and Georgia M. Beasley, MD \\ Department of Surgery, Duke University, Durham, NC
}

Since publication of the German Dermatologic Cooperative Oncology Group-Sentinel Lymph Node Trial (DeCOG-SLT) and the Multicenter Selective Lymphadenectomy Trial II (MSLT-II) in the United States within the last 5 years (2016 and 2017, respectively), completion lymph node dissection (CLND) is no longer routinely performed for patients presenting with primary cutaneous melanoma and a positive sentinel lymph node (SLN) as both studies failed to demonstrate improvements in melanoma-specific survival for patients undergoing CLND versus active surveillance. ${ }^{1,2}$ Furthermore, the 5-year recurrence-free survival (RFS) was similar between groups in updated results from the DeCOG-SLT trial. In contrast, the 3-year disease-free survival (DFS) reported in MSLT-II was greater in the CLND group than in the active surveillance group $(68 \pm 1.7 \%$ vs $63 \pm 1.7 \%, P=0.05)$; the lower DFS in the active surveillance group was largely due to nodal basin recurrences. ${ }^{1,3}$ Together, these studies indicate that isolated primary nodal basin relapses do not impact overall melanoma-specific survival. However, recognition of patients at high risk of nodal recurrence after a positive SLN, and the potential to prevent nodal basin relapses after a positive SLN remain important to providers and are certainly important to patients.

In this issue of Annals of Surgical Oncology, Mitra et al. describe outcomes in 215 patients with a positive SLN who were managed with active surveillance and adjuvant

(C) Society of Surgical Oncology 2021

First Received: 19 February 2021

Accepted: 20 February 2021;

Published Online: 12 March 2021

G. M. Beasley, MD

e-mail: georgia.beasley@duke.edu systemic therapy (47\% of patients), and no CLND. ${ }^{4}$ At 20 months median follow-up, isolated SLN basin recurrences were the most common first site of recurrent disease occurring in $10 \%(n=22 / 215)$ of patients. On multivariate analysis, lymphovascular invasion, the presence of 2 or more involved nodes, and $>1 \mathrm{~mm}$ nodal deposit were associated with higher rates of nodal relapse. In contrast, isolated nodal basin recurrences were reported in 63/820 $(7.7 \%)$ of the nodal observation group in MSLT-II. In MSLT-II, only $33.2 \%$ of patients had SLN disease $>1 \mathrm{~mm}$ compared with $50.2 \%$ of patients in the Mitra et al. study. Only $7 \%$ of patients in DeCOG had SLN disease $>2 \mathrm{~mm}$, and patients with SLN extracapsular extension (EC) were excluded from DeCOG whereas 10\% $(n=21)$ of patients had EC in the Mitra et al. study. This difference in study populations, namely the relatively increased SLN burden in the Mitra et al. study may explain the higher rates of isolated nodal recurrence seen. SLN disease burden has long been shown to be associated with non-sentinel lymph node involvement. ${ }^{5}$ As such, patients should be counseled differently about the risks of developing nodal recurrence based on SLN disease burden, as not all patients with a positive SLN have the same risk of nodal recurrence. Indeed, patients with minimal disease burden in the SLN (the large majority of patients in DeCOG and MSLT-II) had a predicted and actual lower risk of isolated nodal recurrence compared with patients with increased SLN burden. Therefore, providers should emphasize intense surveillance of the nodal basin, especially in patients with increased SLN burden, and patients with increased SLN burden should be advised that the risk for isolated node basin recurrence may approach $10 \%$. For patients who do develop isolated primary node basin recurrence, therapeutic lymph node dissection can then be considered. Rarely are isolated node basin recurrences not amenable to 
surgical resection, which may be a component of a multidisciplinary approach to patients with isolated nodal basin recurrences.

While $22 / 215(10 \%)$ of recurrences occurred in the lymph node basin only, 35 (16\%) had a combination of nodal plus distant, non-nodal locoregional, and/or distant metastasis. Furthermore 13\% $(n=28)$ of patients died during the study with half of those deaths $(n=14)$ attributed to melanoma. Patients with increasing SLN disease burden are not only at increased risk for nodal recurrence compared with patients with a small disease burden in the SLN, but larger SLN disease burden combined with unfavorable primary tumor characteristics can also put patients at increased risk of distant metastases. In the last 5 years, concurrent with changes in nodal basin management, multiple adjuvant systemic therapies have now been approved for patients with SLN positive $(>1 \mathrm{~mm})$ disease based on results of prospective randomized clinical trials demonstrating improvements in recurrence-free survival for patients who receive therapy. ${ }^{6,7}$ For example, in a recently reported update of KEYNOTE-54 at the American Society of Clinical Oncology in 2020, pembrolizumab compared with placebo significantly prolonged distant metastasis-free survival (3.5-year DMFS rate: 64 vs 44\%; HR 0.56, 95\% CI 0.47-0.68) in patients with AJCC version 7 stage IIIA, B, and C melanoma at 3 years of follow up. In the Mitra et al. study, 102 of $215(47 \%)$ patients with a positive SLN received adjuvant therapy. Surprisingly, receipt of adjuvant therapy did not appear to improve disease-free survival, which was $76 \%$ at 18 months for the adjuvant therapy group and $74 \%$ at 2 years for the study group as a whole. This could be due to the retrospective nature of the study and selection bias; only $54 \%$ of patients in the Mitra et al. study that met inclusion criteria for KEYNOTE-54 and COMBI-AD received adjuvant therapy. Additionally, all recently completed adjuvant therapy studies also required CLND prior to trial entry, so it is also possible that in the Mitra et al. study, the 10\% $(n=22)$ of patients with isolated nodal relapse contributed to the higher recurrence rate in the adjuvant therapy group. Notably, the distant disease control for the entire group in the Mitra et al. study was $86 \%$ compared with a distant disease control rate of $94 \%$ of patients in those receiving adjuvant therapy; no direct comparison was made but it is possible that systemic adjuvant therapy did improve distant metastasis-free survival which would be consistent with what has been reported in multiple prospective randomized trials with modern and effective systemic melanoma therapies.

While the potential for increased isolated nodal basin recurrence after a positive SLN and no CLND does not appear to affect overall survival (MSLT-II), the occurrence of distant metastasis, or stage IV disease does negatively impact survival. Adjuvant therapy has demonstrated significant decreases in distant metastasis-free survival and while overall survival data are pending for adjuvant immune therapies, decreases in distant metastasis-free survival are much more likely to impact overall survival. Therefore, patients with increased SLN burden who remain at risk for distant metastasis should be strongly considered for adjuvant therapy, which significantly improves distant metastasis-free survival. There is also ongoing work to improve patient selection for adjuvant therapy so that patients at low risk for the development of distant metastasis can safely avoid adjuvant therapy.

Although isolated nodal recurrence may not impact overall survival, cancer recurrence for patients can be extremely distressing. The benefits of reducing both nodal and distant recurrence and never having to say the words "your cancer is back" can be very powerful for patients. Efforts to prevent recurrence in these patients need to be balanced with the risks and toxicities of therapies, including additional surgery. CLND does not eliminate isolated nodal recurrence, with $1.3 \%$ (10 of 744) of patients in MSLT-II experiencing isolated nodal relapse in the immediate CLND arm. Lymphedema was observed in $24 \%$ of patients in the CLND group compared with $6.3 \%$ in the active surveillance group in MSLT-II. Therefore, use of aggressive surgery with potential permanent morbidity that does not impact overall survival is not warranted for the vast majority of patients. Mitra et al. have proposed a study examining the role of adjuvant nodal radiation and immunotherapy for patients with increased SLN burden ( $\geq 2$ lymph nodes or $>0.5 \mathrm{~mm}$ ) to try to reduce nodal basin recurrences. Further investigation into reducing the risk of nodal recurrence should focus on patients with positive SLN and higher SLN disease burden who are at higher risk of both nodal and systemic recurrence. The rate of isolated nodal recurrences in patients with higher SLN disease burden suggested by this "real world" study is $10 \%$ $(n=22 / 215)$, slightly higher than that seen in MSLT-II (7.7\%), although patients in MSLT-II had a lower disease burden in the SLN compared with the present study population. Physicians should incorporate SLN disease burden into the discussion with the patient and SLN disease burden in combination with primary tumor characteristics should be utilized to further delineate surveillance and adjuvant therapy recommendations.

DISCLOSURES Georgia Beasley: Clinical trial funding paid to institution: Istari oncology, delcath systems, Oncosec, Checkmate Therapeutics Advsiory board Regeneron 2019.

\section{REFERENCES}

1. Faries MB, Thompson JF, Cochran AJ, Andtbacka RH, Mozzillo N, Zager JS, Jahkola T, Bowles TL, Testori A, Beitsch PD, 
Hoekstra HJ, Moncrieff M, Ingvar C, Wouters M, Sabel MS, Levine EA, Agnese D, Henderson M, Dummer R, Rossi CR, Neves RI, Trocha SD, Wright F, Byrd DR, Matter M, Hsueh E, MacKenzie-Ross A, Johnson DB, Terheyden P, Berger AC, Huston TL, Wayne JD, Smithers BM, Neuman HB, Schneebaum S, Gershenwald JE, Ariyan CE, Desai DC, Jacobs L, McMasters KM, Gesierich A, Hersey P, Bines SD, Kane JM, Barth RJ, McKinnon G, Farma JM, Schultz E, Vidal-Sicart S, Hoefer RA, Lewis JM, Scheri R, Kelley MC, Nieweg OE, Noyes RD, Hoon DSB, Wang HJ, Elashoff DA, Elashoff RM. Completion dissection or observation for sentinel-node metastasis in Melanoma. $N$ Engl J Med. 2017;376:2211-22.

2. Leiter U, Stadler R, Mauch C, Hohenberger W, Brockmeyer N, Berking C, Sunderkötter C, Kaatz M, Schulte K-W, Lehmann P, Vogt T, Ulrich J, Herbst R, Gehring W, Simon J-C, Keim U, Martus P, Garbe C. Complete lymph node dissection versus no dissection in patients with sentinel lymph node biopsy positive melanoma (DeCOG-SLT): a multicentre, randomised, phase 3 trial. Lancet Oncol. 2016;17:757-67.

3. Leiter U, Stadler R, Mauch C, Hohenberger W, Brockmeyer NH, Berking C, Sunderkötter C, Kaatz M, Schatton K, Lehmann P, Vogt T, Ulrich J, Herbst R, Gehring W, Simon JC, Keim U, Verver D, Martus P, Garbe C. Final analysis of DeCOG-SLT trial: no survival benefit for complete lymph node dissection in patients with melanoma with positive sentinel node. $J$ ClinOncol. 2019;37:3000-8.
4. Mitra D, Ologun G, Keung EZ et al. Nodal recurrence is a primary driver of early relapse for patients with sentinel lymph node positive melanoma in the modern therapeutic era. Ann Surg Oncol. 2021. https://doi.org/10.1245/s10434-021-09804-3.

5. Dewar DJ, Newell B, Green MA, Topping AP, Powell BW, Cook MG. The microanatomic location of metastatic melanoma in sentinel lymph nodes predicts nonsentinel lymph node involvement. J ClinOncol. 2004;22:3345-9.

6. Eggermont AMM, Blank CU, Mandala M, Long GV, Atkinson V, Dalle S, Haydon A, Lichinitser M, Khattak A, Carlino MS, Sandhu S, Larkin J, Puig S, Ascierto PA, Rutkowski P, Schadendorf D, Koornstra R, Hernandez-Aya L, Maio M, van den Eertwegh AJM, Grob JJ, Gutzmer R, Jamal R, Lorigan P, Ibrahim N, Marreaud S, van Akkooi ACJ, Suciu S, Robert C. Adjuvant Pembrolizumab versus placebo in resected stage III melanoma. $N$ Engl $J$ Med. 2018;378:1789-801.

7. Kwak M, Farrow NE, Salama AKS, Mosca PJ, Hanks BA, Slingluff CL, Beasley GM. Updates in adjuvant systemic therapy for melanoma. J SurgOncol. 2019;119:222-31.

Publisher's Note Springer Nature remains neutral with regard to jurisdictional claims in published maps and institutional affiliations. 